\title{
The risk of metachronous cancers in patients with small-intestinal carcinoid tumors: a US population-based study
}

\author{
Sunil Amin ${ }^{1}$, Richard R P Warner ${ }^{1,2}$, Steven H Itzkowitz ${ }^{1,2}$ and \\ Michelle Kang Kim $^{1,2}$
}

\begin{abstract}
${ }^{1}$ Department of Internal Medicine and ${ }^{2}$ Division of Gastroenterology, Mount Sinai School of Medicine, 5 East 98th Street, 11th Floor, New York, New York 10029, USA

(Correspondence should be addressed to M K Kim at Division of Gastroenterology, Department of Internal Medicine, Mount Sinai School of Medicine; Email: michelle.kim@ mountsinai.org)
\end{abstract}

\begin{abstract}
Small-intestinal carcinoids (SIC) are the most common small-bowel malignancies. We sought to determine the risk of developing SIC before and after other primary malignancies (PM) and the prognosis of patients with SIC, with and without another PM. We used the Surveillance, Epidemiology, and End Results database to identify patients diagnosed with SICs between 1973 and 2007. Multiple primary-standardized incidence ratios were calculated as an approximation of relative risk (RR) to explore the association of SICs with metachronous malignancies. Survival analysis was performed using Kaplan-Meier methods and Cox proportional-hazard models. Among 8331 patients with SICs, $2424(29 \%)$ had another PM at some time. The most common sites were prostate $(26.2 \%)$, breast $(14.3 \%)$, colon $(9.1 \%)$, lung/bronchus $(6.3 \%)$, and bladder (5.3\%). Overall, $67 \%$ of patients had a PM diagnosed before SIC (pre-SIC), 33\% after SIC (postSIC), and $8 \%$ had a PM both before and after SIC. Among the pre-SIC group, the risk of future SIC was increased after cancers of the small bowel (RR 11.86 (95\% Cl: 6.13-20.72)), esophagus (4.05 (1.10-10.36)), colon (1.39 (1.05-1.81)), kidney (1.93 (1.12-3.09)), prostate (1.38 $(1.17-1.62))$, and leukemia $(2.15(1.18-3.61))$. Among the post-SIC group, there was an increased risk of future PM of the small bowel (8.78 (4.54-15.34)), liver (2.49 (1.08-4.91)), prostate (1.25 (1.0-1.53)), and thyroid (2.73 (1.10-5.62)). Compared to patients with only SIC, those with a PM pre-SIC had worse mean survival (57.9 vs 40.9 months, HR 1.55 (1.42-1.69), $P<0.001)$. In conclusion, almost one-third of patients with SICs have an associated metachronous primary tumor. When these primaries occur prior to (but not after) the SIC diagnosis, the prognosis is worse than with an initial SIC. The type of malignancies associated with SICs may guide future screening efforts.
\end{abstract}

Endocrine-Related Cancer (2012) 19 381-387

\section{Introduction}

Small-intestinal carcinoids (SIC) are the most common small-bowel malignancies, representing $31-41 \%$ of all small-bowel neoplasms (DiSario et al. 1994, Lepage et al. 2006, Hatzaras et al. 2007, Bilimoria et al. 2009). Although they may be diagnosed at any time, peak incidence occurs between the ages of 50 and 60 years. SICs are generally characterized by a relatively indolent course, and may produce a number of clinical manifestations. SIC patients may present with or develop metastatic disease.
It is difficult to predict survival patterns in patients with SICs due to their heterogeneous biological behavior (Modlin et al. 2008). The Surveillance, Epidemiology, and End Results (SEER) database predicts 5-year survival as broadly as $43-96 \%$ based on disease extent, tumor site, and grade (Landry et al. 2008). Perhaps more importantly, this survival rate has not changed over time after adjusting for patient demographics, tumor characteristics, and treatment approach (Bilimoria et al. 2009). Recent efforts have attempted to understand the long-term survival and 
correct staging of the disease. Initial attempts at staging were published by the European Neuroendocrine Tumor Society (ENETS) investigators in 2006/2007 and have since been revised (Rindi et al. 2006, 2007, Klöppel et al. 2009). The North American Neuroendocrine Tumor Society (NANETS) has also now published similar guidelines, as have other independent investigators (Landry et al. 2008, Boudreaux et al. 2010, Edge et al. 2010).

Multiple primary cancers in an individual may suggest a genetic predisposition or common environmental risk factors, and understanding the risks of developing neoplasms may guide future screening and surveillance efforts (Neugut \& Robinson 1992). With regard to SICs, metachronous cancers may be a consequence of bioactive, potentially neoplasiapromoting secretory products affecting a variety of cell types (Modlin et al. 2003). Earlier studies investigating the incidence of additional primary cancers among patients with neuroendocrine tumors (NETs) have been either autopsy studies or limited by small sample sizes (Alexander \& Altemeier 1968, Gerstle et al. 1995, Habal et al. 2000). As such, they have focused on gastrointestinal NETs in general, as opposed to more specifically SIC tumors. Most recently, Kamp et al. (2012) published a review of 459 Dutch patients with NETs of the digestive tract and concluded that $13.7 \%$ of patients had a second primary cancer. Although a population-based study by Zar et al. (2008) used the Swedish Cancer Registry to estimate excess risk associated with second primary malignancies (PM) among 3741 SIC patients, no study has used the US-based SEER database to quantify risk, none has investigated the predictors of additional malignancies in SIC patients, and none has utilized survival data to study differences in prognosis between SIC patients with and without additional PM.

The aim of our study was to use the populationbased SEER database, which covers $\sim 28 \%$ of the US population, to follow-up on a large number of SIC patients over time. We sought to investigate not only the risk of developing SIC before and after other PM, but also to assess the prognosis of patients with SIC, with and without another PM, and finally to determine the predictors of additional PM among SIC patients.

\section{Materials and methods}

The Surveillance, Epidemiology, and End-Results (SEER) Program was used to perform a review of patients diagnosed with SIC tumors between January 1, 1973 through December 31, 2007 (National Cancer Institute DCCPS Surveillance Research Program
2009). Using SEER*Stat software (National Cancer Institute, DCCPS, Surveillance Research Program SEER*Stat software (www.seer.cancer.gov/seerstat) version 6.5.2), patients were initially selected based on site codes C170-173, and C178-C179 for the small intestine. We then narrowed our search via ICD-O3 histology codes $8240,8241,8243$, and 8249 to select specifically for SICs. We excluded diagnoses that were noted solely on the death certificate or at autopsy. A latency period of 6 months was used to reduce the possibility of a pre-existing or incidental tumor being discovered during the initial workup of the SIC.

Within the cohort above, the incidence of additional primary cancers, both before and after the diagnosis of small-bowel carcinoid, was determined. Multiple primary-standardized incidence ratios (MP-SIR) were calculated as an approximation of relative risk (RR) to explore the association of SICs with metachronous malignancies. Specifically, we calculated the cumulative age-, sex-, and race-specific person years at risk for each patient and multiplied these by the incidence rate of SICs (for the pre-SIC group) and the incidence rate of various PM (post-SIC) as resulted by the SEER database over the study period to obtain the expected number of SICs or PM. We then computed SIRs by dividing the observed number of each cancer by the expected number of the same cancer (observed/ expected). We calculated $95 \%$ confidence intervals using the Byar approximation to the exact Poisson Test (Breslow \& Day 1987).

Kaplan-Meier (K-M) methods and Cox Proportional-Hazard (CPH) models were used to test survival differences among SIC patients with and without another PM. Multivariate logistic regression was used to predict the factors associated with risk of a future (non-SIC) malignancy. Statistical significance was accepted at the $P<0.05$ level. SAS v9.2 (Carey, $\mathrm{NC}$, USA) was used for all statistical analyses.

\section{Results}

\section{Patient characteristics and demographics}

We identified 8331 individuals diagnosed with an SIC between January 1, 1973 and December 31, 2007 who met our eligibility criteria (Table 1). The age of patients ranged from 14 to 98 years, with a mean of $64.7 \pm 13.4$ years. The majority, $83.3 \%$ of patients were Caucasian, $13.9 \%$ were African-American, and $2.8 \%$ were of other races. Of those diagnosed, $52.7 \%$ of patients were male. The number of SICs diagnosed per decade increased over time, from $6.3 \%$ in $1973-1980$ to $51.4 \%$ during $2000-2007$. Of these, $38.3 \%$ of cases 
Table 1 Demographic characteristics of SIC cohort

\begin{tabular}{lc}
\hline $\begin{array}{l}\text { Demographic } \\
\text { characteristic }\end{array}$ & $\begin{array}{c}\text { Overall cohort } \\
(n=8331)\end{array}$ \\
\hline Mean age (years, s.D.) & $64.7(13.4)$ \\
Race & \\
Caucasian & $6893(82.7 \%)$ \\
African-American & $1149(13.8 \%)$ \\
Other & $231(2.8 \%)$ \\
Unknown & $58(0.8 \%)$ \\
Gender & \\
Male & $4392(52.7 \%)$ \\
Female & $3939(47.3 \%)$ \\
Marital status & \\
Single & $895(10.7 \%)$ \\
Married & $5060(60.7 \%)$ \\
Separated/divorced & $705(8.5 \%)$ \\
Widowed & $1283(15.4 \%)$ \\
Unknown & $388(4.7 \%)$ \\
Year of diagnosis & \\
1973-1980 & $527(6.3 \%)$ \\
1980-1989 & $1088(13.1 \%)$ \\
1990-1999 & $2433(29.2 \%)$ \\
2000-2007 & $4283(51.4 \%)$ \\
SEER historic stage & \\
Localized & $3017(36.2 \%)$ \\
Regional & $2874(34.5 \%)$ \\
Distant & $1979(23.8 \%)$ \\
Unknown & $461(5.5 \%)$ \\
Additional primary malignancy & \\
None & $5907(70.9 \%)$ \\
Before SIC & $1734(20.8 \%)$ \\
After SIC & $890(10.7 \%)$ \\
Both & $200(2.4 \%)$ \\
\hline &
\end{tabular}

were classified as localized disease, $36.5 \%$ as regional, and $25.2 \%$ as distant, using the SEER historic staging criteria.

\section{Incidence and risk of additional primary cancers}

Of the 8331 patients with SICs, 5907 (71\%) had only an SIC without any other malignancy, whereas 2424 (29\%) had an additional PM at some time. The most common sites of additional PM were prostate (26.2\%), breast $(14.3 \%)$, colon $(9.1 \%)$, lung/bronchus $(6.3 \%)$, and bladder (5.3\%) (Table 2).
Two-thirds (67\%) of SIC patients with second cancers had their additional malignancy diagnosed before the SIC (defined herein as pre-SIC), whereas $33 \%$ had additional PM diagnosed after the SIC (postSIC). Among all SIC patients, $8 \%$ of patients had an additional PM both before and after the SIC. There was no difference in the proportion of additional primary cancers among patients with duodenal, jejunal, or ileal SICs ( $30 \%$ vs $28 \%$ vs $32 \%, P=0.14)$.

Among the pre-SIC group, the risk of future SIC was increased after cancers of the small bowel (RR 11.86 (95\% CI: 6.13-20.72)), esophagus (RR 4.05 (1.1010.36)), colon (RR 1.39 (1.05-1.81)), kidney (RR 1.93 (1.12-3.09)), prostate (RR 1.38 (1.17-1.62)), and leukemia (RR 2.15 (CI: 1.18-3.61)). Among the post-SIC group, there was an increased risk of future PM of the small bowel (RR 8.78 (4.54-15.34)), liver (RR 2.49 (1.08-4.91)), prostate (RR 1.25 (1.0-1.53)), and thyroid (RR 2.73 (1.10-5.62)). SIC patients had a decreased risk of future lung cancer (RR 0.60 (0.410.83)) (Tables 3 and 4).

\section{Logistic regression analysis}

Logistic regression analysis was performed to investigate the factors predictive of a future non-SIC primary cancer among patients with SICs (Table 5). Controlling for significant co-factors, lower-stage SIC (regional vs localized OR 0.89, 95\% CI: 0.74-1.08, distant vs localized OR 0.58 , 95\% CI: $0.46-0.73$, $P<0.001)$, increasing age at SIC diagnosis $(51-70$ vs $<51$ years old; OR $1.80,95 \%$ CI: $1.37-2.36,>70$ vs $<51$ years old; OR 2.01, 95\% CI: $1.50-2.70$, $P<0.001$ ), male sex (OR 0.84, 95\% CI: $0.70-1.00$, $P=0.049)$ ), and more recent SIC diagnosis $(1980$ 1989 vs <1980; OR 0.96, 95\% CI: 0.69-1.33, 19901999 vs < 1980; OR 0.70, 95\% CI: $0.51-0.95,>2000$ vs $<1980$ OR 0.39 , 95\% CI: $0.29-0.53, P<0.001)$ were all associated with increased odds of developing a future PM.

Table 2 Most common additional cancers among SIC cohort

\begin{tabular}{|c|c|c|c|c|c|c|}
\hline \multirow[b]{2}{*}{ Additional primary } & \multicolumn{2}{|c|}{ Overall $(n=920)$} & \multicolumn{2}{|c|}{ Pre-SIC $(n=542)$} & \multicolumn{2}{|c|}{ Post-SIC $(n=378)$} \\
\hline & $n$ & $\%$ & $n$ & $\%$ & $n$ & $\%$ \\
\hline Prostate & 241 & 26.2 & 149 & 27.5 & 92 & 24.3 \\
\hline Breast & 132 & 14.3 & 91 & 16.8 & 41 & 10.9 \\
\hline Colon & 84 & 9.1 & 55 & 10.1 & 29 & 7.7 \\
\hline Lung/bronchus & 58 & 6.3 & 24 & 4.4 & 34 & 9.0 \\
\hline Bladder & 49 & 5.3 & 31 & 5.7 & 18 & 4.8 \\
\hline Other & 356 & 38.6 & 192 & 35.5 & 164 & 43.4 \\
\hline
\end{tabular}


Table 3 Cancers associated with increased risk of future SIC (pre-SIC group)

\begin{tabular}{lrrrrrr}
\hline Site & Persons & $\begin{array}{c}\text { Person years at } \\
\text { risk }\end{array}$ & Observed & Expected & RR (O/E) & 95\% Cl \\
\hline Small intestine & 6996 & 38371.55 & 12 & 1.01 & 11.86 & $6.13-20.72$ \\
Esophagus & 16764 & 32441.84 & 4 & 0.99 & 4.05 & $1.10-10.36$ \\
Colon & 200424 & 1343945.75 & 55 & 39.57 & 1.39 & $1.05-1.81$ \\
Kidney & 50596 & 343467.56 & 17 & 107.95 & 1.93 & $1.12-3.09$ \\
Prostate & 396941 & 2631473.74 & 149 & 6.51 & 1.38 & 2.15 \\
Leukemia & 58895 & 335966.60 & 14 & & $1.18-3.61$ \\
\hline
\end{tabular}

\section{Survival analysis}

Multiple survival analyses were performed. Compared to patients with only SIC, those in the pre-SIC group had a worse mean survival (57.9 vs 40.9 mos), both by $\mathrm{K}-\mathrm{M}$ methods $(P<0.001)$ and $\mathrm{CPH}$ models $(\mathrm{HR} 1.55$ (1.420-1.683), $P<0.001)$, controlling for age, year of diagnosis, race, sex, marital status, and stage (Fig. 1). Compared to patients with only SIC, patients with an additional PM before and after the SIC had worse survival by $\mathrm{K}-\mathrm{M}$ methods $(P<0.001)$, but not when controlling for other variables $(P=0.229)$. This finding is likely an artifact, biased by patients with SICs who survive long enough to have a subsequent cancer. Patients in the post-SIC group did not have significantly different survival by $\mathrm{K}-\mathrm{M}$ methods.

\section{Discussion}

Metachronous neoplasia may reflect a genetic predisposition, common environmental exposures or behavioral risk factors, exogenous mitogenic effects of secretory products from a primary tumor causing neoplastic transformation, or a combination of all these factors (Neugut \& Robinson 1992, Modlin et al. 2003). Investigators have previously studied the co-existence of additional primary cancers in carcinoid patients, but only one study has used a populationbased data set, and none has quantified the risk of both future primaries after SIC (post-SIC) as well as the risk of future SIC among patients with non-SIC primaries (pre-SIC) (Zar et al. 2008). Our analysis constitutes not only the first US population-based quantification of the risk of additional cancers among a large cohort of SIC patients, but also investigates the predictors of additional malignancies in SIC patients, and utilizes survival data to study differences in prognosis between SIC patients with and without additional PM.

In our series, almost one-third (29\%) of patients with SICs had an associated metachronous primary tumor, a finding consistent with what other investigators have reported for carcinoid tumors in general. Most recently, Modlin et al. used the SEER database to show that carcinoid tumors of all types were associated with other non-carcinoid tumors in $22.4 \%$ of patients; however, other studies have reported associations with rates varying from 13.7 to $40.7 \%$ (Berge \& Linell 1976, Saha et al. 1989, Marshall \& Bodnarchuk 1993, Kamp et al. 2012). In the majority of these studies, the most common site of additional malignancy was the gastrointestinal tract, a finding not entirely reproduced by our data. While $20.7 \%$ of our additional primaries did occur in the gastrointestinal tract, $26.2 \%$ occurred in the prostate. Furthermore, breast malignancies accounted for $14.3 \%$ of additional primaries. These observations are consistent with a recently published study of a Dutch cohort and likely reflect the trend toward increased cancer surveillance over the last two decades combined with the fact that breast, prostate, lung, and colon cancers are among the most common cancers diagnosed in the U.S (Kamp et al. 2012). Nevertheless, exogenous mitogenic effects of secretory products from a primary tumor causing neoplastic transformation as well as common environmental exposures or behavioral risk factors among a large number of cancers must also be considered (Neugut \& Robinson 1992, Modlin et al. 2003). Increased survival

Table 4 Cancers associated with increased risk after SIC (post-SIC group)

\begin{tabular}{lcccccc}
\hline Site & Persons & $\begin{array}{c}\text { Person years at } \\
\text { risk }\end{array}$ & Observed & Expected & RR (O/E) & $\mathbf{9 5 \% ~ C l ~}$ \\
\hline Small intestine & 3347 & 22390.26 & 12 & 1.37 & 8.78 & $4.54-15.34$ \\
Liver & 3347 & 23390.26 & 8 & 3.21 & 2.49 & $1.08-4.91$ \\
Prostate & 3347 & 23390.26 & 92 & 73.80 & 1.25 & $1.00-1.53$ \\
Thyroid & 3347 & 23390.26 & 7 & 2.57 & 2.73 & $1.10-5.62$ \\
\hline
\end{tabular}


Table 5 Predictors of future primary malignancy among patients with SICs

\begin{tabular}{lllc}
\hline & Odds ratio & $95 \% \mathbf{C l}$ & $\boldsymbol{P}$ value \\
\hline $\begin{array}{l}\text { Sex } \\
\text { Male }\end{array}$ & Reference & & \\
Female & 0.84 & $0.70-1.00$ & \\
Age & & & \\
$<51$ & Reference & & $<0.001$ \\
$51-70$ & 1.80 & $1.37-2.36$ & \\
$>70$ & 2.01 & $1.50-2.70$ & \\
Year of SIC diagnosis & & \\
$<1980$ & Reference & & \\
$1980-1989$ & 0.96 & $0.69-1.33$ & \\
$1990-1999$ & 0.70 & $0.51-0.95$ & \\
$>2000$ & 0.39 & $0.29-0.53$ & \\
Stage of SIC & & & $<0.001$ \\
Localized & Reference & & \\
Regional & 0.89 & $0.74-1.08$ & \\
Distant & 0.58 & $0.46-0.73$ & \\
\hline
\end{tabular}

and subsequent follow-up for the detection of additional malignancies is a less likely explanation, as the survival rate of SICs has not changed over this time period (Bilimoria et al. 2009).

More important than the absolute percentages of associated malignancies is the risk associated with their development among SIC patients. Our data suggest an increased risk of subsequent SIC after certain gastrointestinal malignancies, in particular small bowel, esophagus, and colon, with RRs ranging from 1.39 to 11.86 . These findings may represent common genetic mechanisms, or possibly, as mentioned above, neuroendocrine secretory products acting locally and promoting tumorigenesis. Our 6-month latency period should have guarded against pre-existing or incidental SIC being discovered during the initial workup of the non-SIC primary. The increased risk of future SIC after kidney and prostate cancers is not unexpected, as these cancers are so common in the general population. The increased risk of SIC after leukemia is noteworthy, and may perhaps represent an association with therapeutic radiation.

Our study also suggests that patients with SICs are at increased risk for subsequent PM of the small bowel, liver, prostate, and thyroid, with RRs ranging from 1.25 to 8.78. Again, this may be explained by common genetic or environmental risk factors, and possibly even suggest a syndromic association between NETs of the small intestine and other primary cancers. The apparent protective effect of SICs on the development of future lung cancer is curious, and must be investigated further with more dedicated analyses.

The results of our logistic regression analysis investigating the factors associated with the development of future primary cancers among SIC patients are not surprising. Lower-stage SIC, as expected, increased the chances of developing a future non-SIC primary. These SICs follow a more indolent course, and patients survive long enough to develop a second cancer. Similarly, increasing age at SIC diagnosis was also predictive of the development of future non-SIC cancers. Unrelated to their SIC, these older patients are at increased risk for neoplastic processes in general. Earlier year of SIC diagnosis is likely an artifact of the SEER database, as patients diagnosed closer to the study end-point have fewer years of follow-up to develop additional cancers. Male SIC patients were also more likely to develop a subsequent $\mathrm{PM}$, but the corresponding $P$ value of 0.049 barely met statistical significance.

Lastly, our survival analyses suggest that SIC patients diagnosed with a PM before, but not after, their SIC have a worse prognosis than patients diagnosed with SIC as a cancer of first diagnosis (mean survival 57.9 vs 40.9 months). This finding has implications for both patient and provider, and suggests increased screening and surveillance efforts among the most common pre-SIC neoplasms may be a way of increasing survival from SIC. Small-bowel neoplasms must not be overlooked when considering routine cancer screening.

This study has several limitations. First, we assumed perfect follow-up of cancer survivors in the SEER database. In reality, this is not the case. Second, SICs are rare cancers, and the number of patients with multiple primaries, though much larger than the previously reported series, is small. As such, this



Figure 1 Increased mortality when SIC occurs as a subsequent primary cancer compared to SIC as cancer of first diagnosis. SIC as cancer of first diagnosis, solid line; SIC as subsequent primary cancer, dashed line. Log rank test $(P<0.001)$, cox proportional-hazard model (HR 1.55 (1.420-1.683), $P<0.001)$, controlling for age, year of diagnosis, race, sex, marital status, and stage. 
sample size resulted in our calculating SIRs based on small expected values. Although we tried to group anatomic sites together to minimize this effect (e.g. ascending, transverse, and descending colon), this was not possible for some cancer sites. Third, although we used a 6-month latency period to reduce the possibility of pre-existing or incidental tumors being discovered during the workup of the initial primary cancer, we cannot conclude with certainty that the SEER database does not include any redundancy.

In summary, our study constitutes the first US population-based quantification of the risk of additional cancers in SIC patients. We show that almost one-third of SIC patients develop at least one additional PM at some time, and that two-thirds of these additional malignancies occur prior to the SIC diagnosis. For this latter cohort, patients with an additional malignancy prior to their SIC diagnosis, survival is worse than when SIC occurs as a cancer of first diagnosis. Tailoring screening and surveillance efforts based on the type of malignancies associated with SICs may aid in the diagnosis of early-stage disease, and subsequently help to reduce SIC mortality.

\section{Declaration of interest}

The authors declare that there is no conflict of interest that could be perceived as prejudicing the impartiality of the research reported.

\section{Funding}

This research did not receive any specific grant from any funding agency in the public, commercial or not-for-profit sector.

\section{References}

Alexander JW \& Altemeier WA 1968 Association of primary neoplasms of the small intestine with other neoplastic growths. Annals of Surgery 167 958-963. (doi:10.1097/ 00000658-196806000-00017)

Berge T \& Linell F 1976 Carcinoid tumors frequency in a defined population during a 12-year-period. Acta Pathologica et Microbiologica Scandinavica 84 322-330. (doi:10.1111/j.1699-0463.1976.tb00122.x)

Bilimoria KY, Bentrem DJ, Wayne JD, Ko CY, Bennett CL \& Telamonti MS 2009 Small bowel cancer in the United States: changes in epidemiology, treatment, and survival over the last 20 years. Annals of Surgery 249 63-71. (doi:10.1097/SLA.0b013e31818e4641)

Boudreaux JP, Klimstra DS, Hassan MM, Woltering EA, Jensen RT, Goldsmith SJ, Nutting C, Bushnell DL, Caplin ME \& Yao JC 2010 The NANETS consensus guideline for the diagnosis and management of neuroendocrine tumors: well-differentiated neuroendocrine tumors of the Jejunum, Ileum, Appendix, and Cecum. Pancreas 39 753-766. (doi:10.1097/MPA.0b013e3181ebb2a5)

Breslow NE \& Day NE 1987 Statistical methods in cancer research. Volume II: the design and analysis of cohort studies. IARC Scientific Publications 82 1-406.

DiSario JA, Burt RW, Vargas H \& McWhorter WP 1994 Small bowel cancer: epidemiological and clinical characteristics from a population-based registry. American Journal of Gastroenterology 89 699-701.

Edge SB, Byrd DR, Compton CC, et al. (Eds) 2010 In AJCC (American Joint Committee on Cancer) Cancer Staging Manual, pp 181-191, 7th edn. New York, NY, USA: Springer.

Gerstle JT, Kauffman GL Jr \& Koltun WA 1995 The incidence, management, and outcome of patients with gastrointestinal carcinoids and second primary malignancies. Journal of the American College of Surgeons 180 427-432.

Habal N, Sims C \& Bilchik AJ 2000 Gastrointestinal carcinoid tumors and second primary malignancies. Journal of Surgical Oncology 75 310-316. (doi:10.1002/10969098(200012)75:4<306::AID-JSO14>3.0.CO;2-3)

Hatzaras I, Palesty JA, Abir F, Sullivan P, Kozol RA, Dudrick SJ \& Longo WE 2007 Small-bowel tumors: epidemiologic and clinical characteristics of 1260 cases from the Connecticut tumor registry. Archives of Surgery 142 229-235. (doi:10.1001/archsurg.142.3.229)

Kamp K, Damhuis RAA, Feelders RA \& de Herder WW 2012 Occurrence of second primary malignancies in patients with neurodendocrine tumors of the digestive tract and pancreas (GEP-NET). Endocrine-Related Cancer 19 95-99. (doi:10.1530/ERC-11-0315)

Klöppel G, Couvelard A, Perren A, Komminoth P, McNicol AM, Nilsson O, Scarpa A, Scoazec JY, Wiedenmann B, Papotti M et al. 2009 ENETS Consensus Guidelines for the Standards of Care in Neuroendocrine Tumors: towards a standardized approach to the diagnosis of gastroenteropancreatic neuroendocrine tumors and their prognostic stratification. Neuroendocrinology 90 162-166. (doi:10.1159/000182196)

Landry CS, Brock G, Scoggins CR, McMasters KM \& Martin RC II 2008 A proposed staging system for small bowel carcinoid tumors based on an analysis of 6380 patients. American Journal of Surgery 196 896-903. (doi:10.1016/ j.amjsurg.2008.07.042)

Lepage C, Bouvier AM, Manfredi S, Dancourt V \& Faivre J 2006 Incidence and management of primary malignant small bowel cancers: a well-defined French population study. American Journal of Gastroenterology 101 2826-2832. (doi:10.1111/j.1572-0241.2006.00854.x)

Marshall JB \& Bodnarchuk G 1993 Carcinoid tumors of the gut. Journal of Clinical Gastroenterology 16 123-129. (doi:10.1097/00004836-199303000-00009)

Modlin IM, Lye KD \& Kidd M 2003 A 5-decade analysis of 13,715 carcinoid tumors. Cancer 97 934-959.

(doi:10.1002/cncr.11105) 
Modlin IM, Oberg K, Chung DC, Jensen RT, de Herder WW, Thakker RV, Caplin M, Delle Fave G, Kaltsas GA, Krenning EP et al. 2008 Gastroenteropancreatic neuroendocrine tumours. Lancet Oncology 9 61-72. (doi:10.1016/S1470-2045(07)70410-2)

National Cancer Institute, DCCPS, Surveillance Research Program, Cancer Statistics Branch, released April 2009, based on the November 2008 submission. Surveillance, Epidemiology, and End Results (SEER) Program (www.seer.cancer.gov) SEER*Stat Database: Incidence SEER 9 Regs Limited-Use, Nov 2008 Sub (1973-2006) $<$ Katrina/Rita Population Adjustment $>$ - Linked To County Attributes - Total U.S., 1969-2006 Counties. Neugut AI \& Robinson E 1992 Multiple primary neoplasms. Cancer Journal 5 245-248.

Rindi G, Klöppel G, Alhman H, Caplin M, Couvelard A, de Herder WW, Eriksson B, Falchetti A, Falconi M, Komminoth P et al. 2006 TNM staging of foregut (neuro)endocrine tumors: a consensus proposal including a grading system. Virchows Archiv 449 395-401. (doi:10.1007/s00428-006-0250-1)
Rindi G, Klöppel G, Couvelard A, Komminoth P, Körner M, Lopes JM, McNicol AM, Nilsson O, Perren A, Scarpa A et al. 2007 TNM staging of midgut and hindgut (neuro) endocrine tumors: a consensus proposal including a grading system. Virchows Archiv 451 757-762. (doi:10.1007/s00428-007-0452-1)

Saha S, Hoda S, Godfrey R, Sutherland C \& Raybon K 1989 Carcinoid tumors of the gastrointestinal tract: a 44-year experience. Southern Medical Journal 82 1501-1505. (doi:10.1097/00007611-198912000-00011)

Zar N, Garmo H, Holmberg L \& Hellman P 2008 Risk of second primary malignancies and causes of death in patients with adenocarcinoma and carcinoid of the small intestine. European Journal of Cancer 44 718-725. (doi:10.1016/j.ejca.2007.12.003)

Received in final form 12 March 2012 Accepted 15 March 2012 Made available online as an Accepted Preprint 26 March 2012 